\title{
TRANSIENT FLOW THROUGH SOIL INTO OPEN DITCHES WITH CONSTANT RECHARGE
}

\author{
Christos Tzimopoulos* and Stavros Yannopoulos* \\ Department of Rural Engineering \\ *School of Surveying \& Rural Engineering-AUTH. 54006-Thessaloniki, Greece
}

\begin{abstract}
An analytical solution of the linearized Boussinesq equation is presented, which is used to predict the temporal and spatial variations of the groundwater table in a free unconfined aquifer based upon impermeable layer. The variations are caused by the sudden rise of the water table in an adjacent ditch, and the simultaneous constant rainfall or irrigation, uniformly distributed over the soil surface. The results of the presented solution are in very close agreement with those obtained from the numerical solution of the linerarized Boussinesq equation and at time $t \rightarrow \infty$, approach those of the nonlinear problem.
\end{abstract}

\section{INTRODUCTION}

The location of the water table is of great importance for a variety of natural problems that are encountered in nature, for example, the lowering of groundwater level in a desired depth for the optimum development of plants, the determination of safety zones in a contaminated groundwater system and many others.

The groundwater flow is generally nonsteady and is considered to be described satisfactorily by a nonlinear partial differential equation of parabolic type, well-known as the Boussinesq equation. This equation is widely used in most groundwater problems and is derived from the combination of the mass conservation and Darcy laws, based upon the Dupuit-Forchheimer assumptions.

A general analytical solution of the Boussinesq equation subjected to initial and boundary conditions has not yet been found, because of its nonlinearity. Analytical solutions of this equation have been presented by different investigators for specific drainage problems with certain assumptions on the initial and boundary conditions $/ 8 /$. However, the solution of Boussinesq equation has been achieved either by approximate methods (for example, van Schilfgraade /13/) or by finite difference (for example, Tzimopoulos and Terzidis $/ 10 /$ ) and by finite elements methods (for example, Neuman and Witherspoon /7/; Tzimopoulos /11/). 
Analytical solutions of the linearized Boussinesq equation for drainage problem in the case of drain pipes with a constant recharge from rain or irrigation, have been presented by $/ 2-6 /$ and many others.

Dumm /1/ presented a solution of the linear Boussinesq equation, which satisfies the case of sudden lowering of the water table at time $\mathrm{t} \rightarrow \infty$. However, this solution has the disadvantage that for the case of the rising water table it is invalid for time $\mathrm{t} \rightarrow \infty$. Van Giesen et al. /12/ presented an analytical solution of the Laplace equation by expansion in Fourier series for the case of lowering the water table and they compared their solution with that of Dumm $/ 1 /$.

In the present paper the nonsteady flow is studied in an unconfined aquifer of finite length, based upon an impermeable layer. This flow is created from a sudden rise of the water table in an adjacent ditch and the simultaneous constant rain or irrigation, uniformly distributed over the soil surface. The Boussinesq equation is linearized with a special linearization technique and transformed to nondimensional form. The Laplace transformation is used to solve the problem. The results of the solution are in very close agreement with the ones obtained from the linearized problem of the numerical solution and at time $t \rightarrow \infty$ approach those of the nonlinear problem.

\section{MATHEMATICAL FORMULATION}

The problem under consideration is given in Figure 1. At the beginning of the phenomenon the water table in the ditches and the groundwater is considered to be at the same level $h_{0}$ from the horizontal impermeable layer. At the time $\mathrm{t}>0$, a rain $\mathrm{N}$ of constant intensity falls, uniformly distributed over the soil surface, from raindrop or irrigation. This rain is considered to infiltrate entirely into the soil and consequently, there is no surface runoff. Moreover, at the same time, the water table in the ditch of the left rises suddenly at level $h_{1}$. Thus, unsteady groundwater flow starts between the two ditches.

In the problem under consideration, it is assumed that between the two ditches, the soil is

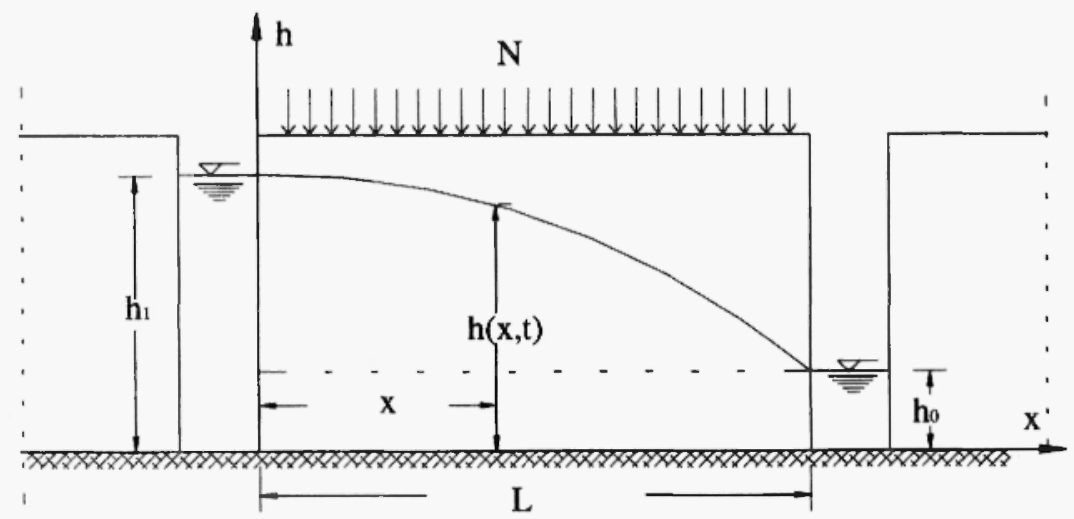

Fig. 1. Definition sketch 
homogeneous and isotropic and that the Dupuit-Forchheimer assumptions are valid.

The movement of groundwater in the flow system under consideration can be represented by the following Boussinesq equation:

$$
\frac{\partial \mathrm{h}}{\partial \mathrm{t}}=\frac{\mathrm{K}}{\mathrm{n}_{\mathrm{e}}} \frac{\partial}{\partial \mathrm{x}}\left(\mathrm{h} \frac{\partial \mathrm{h}}{\partial \mathrm{x}}\right)+\frac{\mathrm{N}}{\mathrm{n}_{\mathrm{e}}}, \quad \mathrm{h}=\mathrm{h}(\mathrm{x}, \mathrm{t})
$$

subject to the initial and boundary conditions :

$$
\begin{array}{lll}
t=0, & 0<x<L, & h(x, 0)=h_{0}, \\
t>0, & x=0, & h(0, t)=h_{1}, \\
t>0, & x=L, & h(L, t)=h_{0},
\end{array}
$$

where $h(x, t)=$ the water table above the horizontal impermeable layer, $x=$ horizontal distance from the origin of the axes, $L=$ spacing between the ditches, $t=$ time of observation, $h_{0}=$ initial water table,$h_{1}=$ steady water table of the left ditch, $N=$ constant raindrop, $n_{e}=$ effective soil porosity and $K=$ hydraulic conductivity.

According to van Schilfgaarde /13/, equations (2.1) can be linearized and written as follows:

$$
\begin{array}{lll}
\frac{\partial h^{2}}{\partial t}=\frac{K B}{n_{e}} \frac{\partial^{2} h^{2}}{\partial x^{2}}+\frac{2 N B}{n_{e}}, & \\
t=0, & h(x, 0)=h_{0} \text { or } \quad h^{2}(x, 0)=h_{0}^{2} \\
t>0, & h(0, t)=h_{1} \quad \text { or } \quad h^{2}(x, 0)=h_{1}^{2} \\
t>0, & h(L, t)=h_{0} \quad \text { or } \quad h^{2}(L, t)=h_{0}^{2}
\end{array}
$$

where $B=\left(h_{1}+h_{0}\right) / 2$ is the constant of the linearization.

It is convenient to write the equations (2.5)-(2.8) in a non dimensional form, by using the following substitutions:

$$
\tau=\frac{k-\underline{n^{2}}}{n_{e} L^{2}} t, \quad X=\frac{x}{L}, \quad H=\frac{h}{h_{l}} .
$$


Then, the problem given by equations $(2.5)-(2.8)$ becomes:

$$
\begin{array}{lll}
\frac{\partial \mathrm{H}^{2}}{\partial \tau}=\frac{\partial^{2} \mathrm{H}^{2}}{\partial \mathrm{X}^{2}}+\varepsilon, & \mathrm{H}=\mathrm{H}(\mathrm{X}, \tau) \\
\tau=0, & 0<\mathrm{X}<1, & \mathrm{H}_{\mathrm{c}}^{2}(\mathrm{X}, 0)=\frac{\mathrm{h}_{\mathrm{o}}^{2}}{\mathrm{~h}_{1}^{2}} \\
\tau>0, & \mathrm{H}_{1}^{2}(0, \tau)=1 \\
\tau>0, & \mathrm{X}=0, & \mathrm{H}_{\mathrm{c}}^{2}(1, \tau)=\frac{\mathrm{h}_{\mathrm{o}}^{2}}{\mathrm{~h}_{1}^{2}}
\end{array}
$$

where $\varepsilon=\frac{2 \mathrm{~N} \mathrm{~L}^{2}}{\mathrm{~K} \mathrm{~h}_{1}^{2}}$

By using the following transformation:

$$
\mathrm{w}=\mathrm{H}^{2}
$$

equations (2.9)-(2.12) are written:

$$
\begin{array}{lll}
\frac{\partial w}{\partial \tau}=\frac{\partial^{2} w}{\partial X^{2}}+\varepsilon, & w=w(X, \tau) \\
\tau=0, & 0 \leq X<1, & w(X, 0)=w_{0}=\frac{h_{0}^{2}}{h_{1}^{2}} \\
\tau>0, & \mathrm{w}(0, \tau)=w_{1}=1 \\
\tau>0, & \mathrm{w}(1, \tau)=w_{0}=\frac{h_{0}^{2}}{h_{1}^{2}}
\end{array}
$$

\section{ANALYTICAL SOLUTION}

\subsection{Solutions for small times}

Applying Laplace transform with respect to $\tau$, to equations (2.14), (2.16) and (2.17) we have:

$$
\bar{w}^{\prime \prime}-s \bar{w}+w_{0}+\frac{\varepsilon}{s}=0
$$

with boundary conditions

$$
\bar{w}(0, s)=\frac{1}{s}
$$




$$
\bar{w}(1, s)=\frac{w_{0}}{s}
$$

where $\bar{w}=£\{w\}$.

The general solution of equation (3.1) is given by:

$$
\bar{w}(X, s)-\frac{w_{0}}{s}-\frac{\varepsilon}{s^{2}}=C_{1} e^{X \sqrt{s}}+C_{2} e^{-X \sqrt{s}}
$$

in which $C_{1}$ and $C_{2}$ are constants, that are determined from the boundary conditions (3.2) and (3.3). Specifically, from the combination of equations (3.2) and (3.4):

$$
C_{i}=-\frac{1-w_{0}}{s} \frac{e^{-\sqrt{s}}}{2 \sinh (\sqrt{s})}-\frac{\varepsilon}{s^{2}} \frac{1-e^{-\sqrt{s}}}{2 \sinh (\sqrt{s})}
$$

and from the combination of equations (3.3) and (3.4):

$$
C_{2}=\frac{1-w_{0}}{s} \frac{e^{\sqrt{s}}}{2 \sinh (\sqrt{s})}+\frac{\varepsilon}{s^{2}} \frac{1-e^{\sqrt{s}}}{2 \sinh (\sqrt{s})}
$$

Substitution of the $C_{1}$ and $C_{2}$ in equation (3.4) yields the following solution for the equation (3.1):

$$
\bar{w}(X, s)=\frac{\bar{w}_{0}}{s}+\frac{\varepsilon}{s^{2}}\left\{1-\frac{\sinh (X \sqrt{s})}{\sinh (\sqrt{s})}-\frac{\sinh \{(1-X) \sqrt{s}\}}{\sinh (\sqrt{s})}\right\}+\frac{1-w_{0}}{s} \frac{\sinh (1-X) \sqrt{s}}{\sinh (\sqrt{s})}
$$

Taking the inverse Laplace transform of equation (3.7):

$$
\begin{aligned}
\frac{w(X, \tau)-w_{0}}{1-w_{0}}= & 1-X+\frac{2}{\pi} \sum_{n=1}^{\infty} \frac{(-1)^{n}}{n} e^{-n^{2} \pi^{2} \tau} \sin \{n \pi(1-X)\} \\
& -\frac{4}{\pi^{3}} \frac{\varepsilon}{1-w_{0}} \sum_{n=1}^{\infty} \frac{(-1)^{n}}{n^{3}}\left(1-e^{-n^{2} \pi^{2} \tau}\right) \sin \left(\frac{n \pi}{2}\right) \cos \left\{n \pi\left(X-\frac{1}{2}\right)\right\}
\end{aligned}
$$

Equation (3.8) is written as follows:

$$
\begin{aligned}
\frac{w(X, \tau)-w_{0}}{1-w_{0}}=1-X & \div \frac{\varepsilon X(1-X)}{2\left(1-w_{0}\right)}+\frac{2}{\pi} \sum_{n=1}^{\infty} \frac{(-1)^{n}}{n} e^{-n^{2} \pi^{2} \tau} \sin \{n \pi(1-X)\} \\
& \div \frac{4}{\pi^{3}} \frac{\varepsilon}{1-w_{0}} \sum_{n=1}^{\infty} \frac{(-1)^{n}}{n^{3}} e^{-n^{2} \pi^{2} \tau} \sin \left(\frac{n \pi}{2}\right) \cos \left\{n \pi\left(X-\frac{1}{2}\right)\right\}
\end{aligned}
$$


because the expansion of $\varepsilon \frac{X}{2}(1-X)$ in Fourier series is:

$\varepsilon \frac{X}{2}(1-X)=-\frac{4 \varepsilon}{\pi^{3}} \sum_{n=1}^{\infty} \frac{(-1)^{n}}{n^{3}} \sin \left(\frac{n \pi}{2}\right) \cos \left\{n \pi\left(X-\frac{1}{2}\right)\right\}$

Equation (3.9) satisfies the initial condition (eq. 2.14) and, also, the boundary conditions (eqs. 2.16 kaı 2.17). Indeed:

Initial condition

For $\tau=0$, equation (3.9) is written:

$$
\begin{aligned}
\frac{w(X, 0)-w_{0}}{1-w_{0}}=1-X & +\frac{\varepsilon X(1-X)}{2\left(1-w_{0}\right)}+\frac{2}{\pi} \sum_{n=1}^{\infty} \frac{(-1)^{n}}{n} \sin \{n \pi(1-X)\} \\
& +\frac{4}{\pi^{5}} \frac{\varepsilon}{i-w_{0}} \sum_{n=1}^{\infty} \frac{(-1)^{n}}{n^{3}} \sin \left(\frac{n \pi}{2}\right) \cos \left\{n \pi\left(X-\frac{1}{2}\right)\right\}
\end{aligned}
$$

The combination of equations (3.9) and (3.11) yields $w=w_{0}$ (equation 2.15) because, for $0 \leq X<1$ :

$$
1-X=-\frac{2}{\pi} \sum_{n=1}^{\infty} \frac{(-i)^{n}}{n} \sin \{n \pi(1-X)\}
$$

\section{Boundary conditions}

For $\tau>0$ and $\mathrm{X}=0$ equation (3.9) is written:

$\frac{w(0, \tau)-w_{0}}{1-w_{0}}=1$

and then, $w(0, \tau)=1$ (equation 2.16)

Similarly, for $\tau>0$ and $X=1$ equation (3.9) is written:

$$
\frac{w(1, \tau)-w_{0}}{1-w_{0}}=0
$$

and then, $w(1, \tau)=w_{0}$ ((equation 2.17).

Consequently, equation (3.9) is the solution of the problem of equations (2.14)-(2.17). 


\subsection{Solution for large times}

For large times $(\tau \rightarrow \infty)$ equation (3.9) takes the form:

$$
\frac{w(X, \infty)-w_{0}}{1-w_{0}}=1-X+\frac{\varepsilon X(1-X)}{2\left(1-w_{0}\right)}
$$

because, for $\tau \rightarrow \infty$ the term $e^{-n^{2} \pi^{2} \tau} \rightarrow 0$ and then:

$$
\mathrm{w}=1-\frac{\varepsilon \mathrm{X}^{2}}{2}-\left(1-\mathrm{w}_{\mathrm{o}}-\frac{\varepsilon}{2}\right) \cdot \mathrm{X}
$$

Equation (3.12) is the solution of the steady state flow of the Boussinesq equation $\frac{d^{2} H^{2}}{d X^{2}}+\varepsilon=0$ with boundary conditions $\mathrm{H}^{2}(0)=1$ and $\mathrm{H}^{2}(1)=\mathrm{H}_{0}{ }^{2}$ and then, constitutes a limit state of balance for $\tau \rightarrow \infty$.

\subsection{Rate of recharge}

The flux $Q(x, t)=Q$ per unit length of the ditch through a vertical plane $x$ can be found by Darcy equation:

$$
Q=-K h \frac{d h}{d x}
$$

The combination of the equations (2.13), (3.9) $\mathrm{k} \alpha \mathrm{l}$ (3.13) yields:

$$
\begin{aligned}
\mathrm{Q}_{\mathbf{X}}^{\prime}=-\frac{\mathrm{K}\left(\mathrm{L}_{1}^{2}-\mathrm{h}_{0}^{2}\right)}{2 \mathrm{~L}}\{ & 1: \frac{\varepsilon(1-2 \mathrm{X})}{2\left(1-\mathrm{w}_{0}\right)}+2 \sum_{n=1}^{\infty}(-1)^{n+1} \mathrm{e}^{-n^{2} \pi^{2} \tau} \cos \{n \pi(1-X)\} \\
& \left.+\frac{4 \varepsilon}{\pi^{2}\left(1-w_{0}\right)} \sum_{n=1}^{\infty} \frac{(-1)^{n+1}}{n^{2}} e^{-n^{2} \pi^{2} \tau} \sin \left(\frac{n \pi}{2}\right) \sin \left\{n \pi\left(X-\frac{1}{2}\right)\right\}\right\}
\end{aligned}
$$

Consequently, the fluxes through the vertical planes $x=0$ and $x=L$, according to equation (3.14), are respectively:

$$
\begin{aligned}
\left.Q\right|_{x=0}=-\frac{K\left(h_{1}^{2}-h_{0}^{2}\right)}{2 L}\{-1 & +\frac{\varepsilon}{2\left(1-w_{0}\right)}+2 \sum_{n=1}^{\infty}(-1)^{2 n+1} e^{-n^{2} \pi^{2} \tau} \\
& \left.+\frac{4 \varepsilon}{\pi^{2}\left(1-w_{0}\right)} \sum_{n=1}^{m} \frac{(-1)^{n+1}}{n^{2}} e^{-n^{2} \pi^{2} \tau} \sin ^{2}\left(\frac{n \pi}{2}\right)\right\}
\end{aligned}
$$


and

$$
\begin{aligned}
\left.Q\right|_{x=L}=-\frac{K\left(h_{1}^{2}-h_{0}^{2}\right)}{2 L}\{-i- & \frac{\varepsilon}{2\left(1-w_{1}\right)}+2 \sum_{n=1}^{\infty}(-1)^{n+1} e^{-n^{2} \pi^{2} \tau} \\
& \left.+\frac{4 \varepsilon}{\pi^{2}\left(1-w_{0}\right)} \sum_{n-1}^{\infty} \frac{(-1)^{n+1}}{n^{2}} e^{-n^{2} \pi^{2} \tau} \sin ^{2}\left(\frac{\pi \pi}{2}\right)\right\}
\end{aligned}
$$

For large times ( $\tau \rightarrow \infty)$, equations (3.15) and (3.16) can be written respectively, as follows:

$$
\mathrm{Q}_{\mathrm{X}=0}^{\prime}=-\frac{\mathrm{K}\left(\mathrm{h}_{1}^{2}-\mathrm{h}_{0}^{2}\right)}{2 \mathrm{~L}}\left\{-1+\frac{\varepsilon}{2\left(1-\mathrm{w}_{0}\right)}\right\}
$$

and

$$
Q_{1 x=L}^{l}--\frac{K\left(h_{1}^{2}-h_{0}^{2}\right)}{2 L}\left\{-i-\frac{E}{2\left(1-w_{0}\right)}\right\}
$$

Equations (3.17) and (3.18) correspond to the steady state flux conditions.

From equation (3.17) the non dimensional flux $Q^{*}$ is:

$$
Q^{*}=1 \cdot \frac{\varepsilon}{2\left(1-w_{0}\right)}
$$

It is easily concluded that the non dimensional flux $Q^{*}$ on the vertical plane $X=0$ varies from $0(t=0)$, until the limiting value of equation (3.19) for $\tau \rightarrow \infty$.

\section{NUMERICAL SOLUTION}

In order to test the results of the analytical solution, the linearized equation (2.14) is solved by the finite difference method. Many computational schemes, as those e.g. of Dufort-Frankel, Douglas, Crank-Nicolson etc. /9/ have been presented in the literature for the solution of the problem. In the present paper, the Laasonen computational scheme is used, which approximates equation (2.14) to the order of $\mathrm{O}(\Delta \tau)+\mathrm{O}\left(\Delta \mathrm{X}^{2}\right)$. This scheme is unconditionally stable $19,10 /$. For the numerical solution of equation (2.14) subject to the conditions (2.15)-(2.17) a similar procedure was applied as it is described by Tzimopoulos and Terzidis $/ 10 /$. 


\section{APPLICATION}

Figure 2 shows the water table variations for $\varepsilon=0.4\left(h_{1}=1\right.$ and $\left.h_{0}=0.3\right)$. The continuous lines correspond to the equation (3.9), while the dashed ones correspond to the numerical solution for different nondimensional times. From this figure, the very close agreement of the two solution is concluded.

Figure 3 represents the non dimensional flux $Q^{*}$ variation with time $\tau$ at the vertical plane $X=0$. It is easily deduced, for large times $(t \rightarrow \infty) Q^{*}$ approaches the limiting value 0.714285 , which results from equation (3.19) for $\varepsilon=0.4$ and $w_{0}=0.3$.

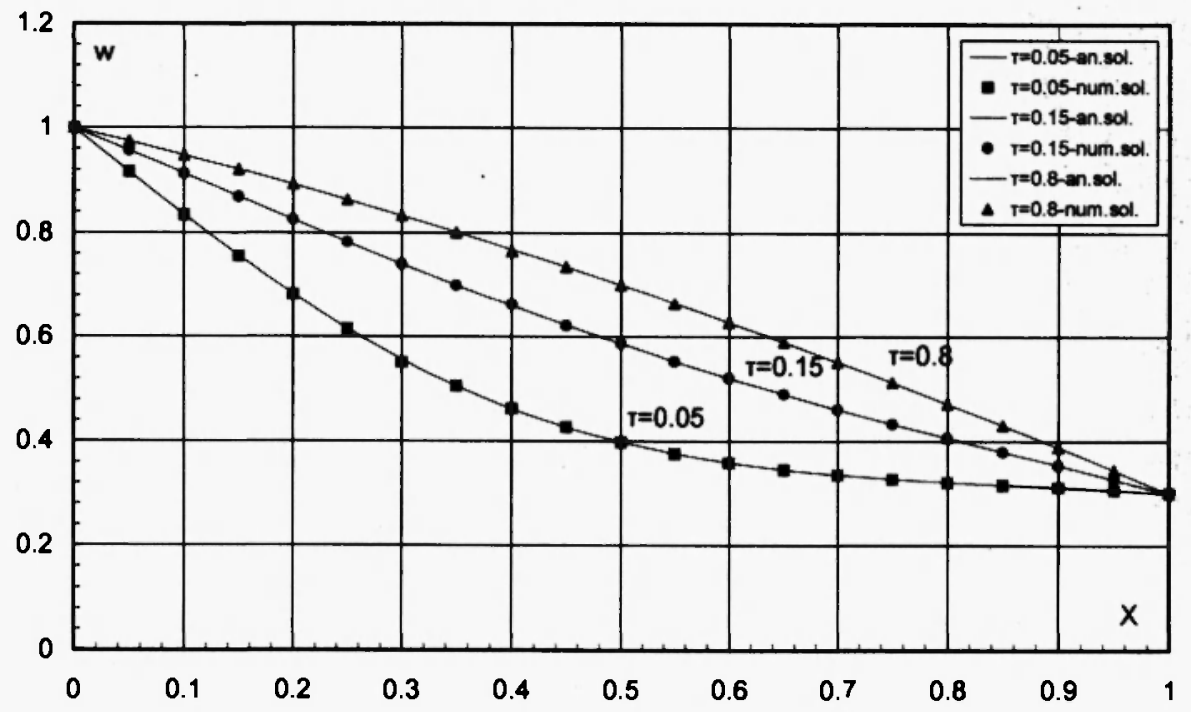

Fig. 2. Water table profiles for different nondimensional times $\tau$.

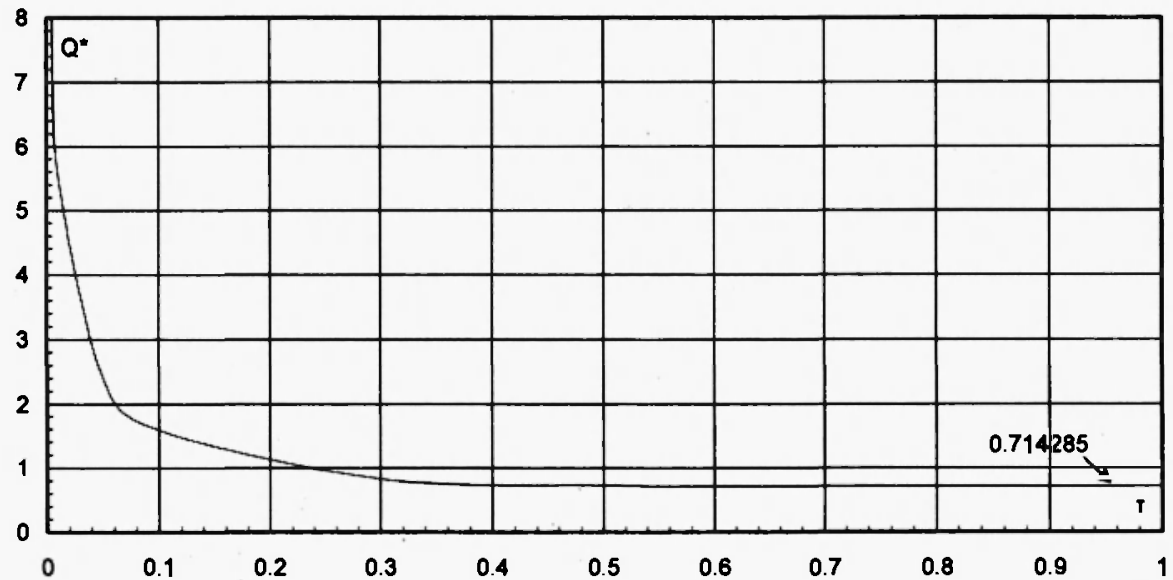

Fig. 3. Variation of the nondimensional flux $\mathrm{Q}^{*}$ with time at the vertical plane $\mathrm{X}=0$. 


\section{CONCLUSIONS}

The presented procedure for finding an analytical solution by the Laplace transform is very general with respect to the specified boundary conditions. The applied linearization technique is proved appropriate because for $t \rightarrow \infty$ the analytical profiles of our solution coincide with those of the non linear Boussinesq equation.

The results of the present solution are in very close agreement with those obtained from the numerical solution of the linearized Boussinesq equation.

It is noted that the use of handheld calculators renders the computational procedures of the analytical solutions easy, in order to study practical problems. The present solution (equation 3.9) can be easily applied to the study of this kind of problems under flux conditions described above.

\section{REFERENCES}

1. L.D. Dumm, Agric. Eng., 35, 726-730 (1954).

2. M.A. Gill., J. Hydrol., 70, 337-352 (1984).

3. D.A. Kraijenhoff van de Leur, De Ingenieur, 40, 87-94 (1958).

4. M. Maasland, M., J. Geophys. Res., 64, 549-559 (1959).

5. M. Marino, J. Hydrol., 23, 289-298 (1974).

6. S. Mustafa, J. Hydrol, 95, 269-276 (1987).

7. S. P. Neuman. and P.A. Witherspoon, Water Resour. Res., 17, 611-623 (1971).

8. P.V. Polubarinova-Kochina, Theory of Groundwater Movement (English translation by R.J.M. De Wiest), Princeton, University Press, Princeton, N.J., 1962; p. 613

9. G. Terzidis, J. Irrig .Drain. Div. Proc., ASCE, No. IR4, 381-389 (1968).

10. C. Tzimopoulos et G.Terzidis, J. Hydrol., 27, 73-93 (1975).

11. C. Tzimopoulos, J. Hydrol., 30, 1-18 (1976).

12. N.C. van de Giesen, J.Y Parlange and T.S. Steenhuis, Water Resour. Res., 30, 3033-3039 (1994).

13. J. van Schilfgaarde, Hydroscience, 6, Ven te Chow (ed.), Academic Press, N.Y., 1970, pp.43-106. 